\title{
OARLCAM: An Ontology-based Approach for Reusing and Learning through Context-aware Annotations Memory
}

\author{
http://dx.doi.org/10.3991/ijet.v9i5.3088
}

\author{
N. Aloui and F. Gargouri \\ University of Sfax, Sfax, Tunisia
}

\begin{abstract}
In this paper, we present an approach to adapt and reuse learning annotations and contexts. Then we explain the process to capitalization and reuse of learning annotations and associated learning contexts. This is to provide to the actor, an appropriate learning which is reviewed or validated previously by others, with similar learning contexts. The modeling and the formalization of learning annotations and learning contexts will allow to define functions for their comparison and their evaluations, in order to reuse them. We propose our method measuring similarities. In this way, we get learning annotations dedicated to a given and well-defined pedagogical goal. An alignment between the two types of ontology, respectively that of the annotation and that of the context will provide us with a learning based on annotations according to the current context which requires a pertinent criterion of similarity between the learning contexts and the learning annotations. This approach allows to manage annotations and adapt actors (learner, tutor, teacher, and coauthor) behaviours with the various contexts of their activities. It offers a great reutilisability to share and have a better quality of learning.
\end{abstract}

Index Terms-learning annotation; context ; Ontology alignement; adaptation, Reuse.

\section{INTRODUCTION}

The information age, particularly the development of new information and communication technologies (ICT), has a huge impact on the way we learn and teach. The integration of ICT in the teaching process has opened new ways for learning, such as distance learning. It is, in fact, the use of new internet and multimedia technologies to improve the quality of training through remote access to resources and services, and thus facilitates collaborations and exchange of knowledge between teachers, learners, tutors, and co-authors.

Different actors in a CEHL (Computing Environment for Human Learning), post subjective, explanatory, analytical, critical and/or prescriptive annotations using different annotation tools. These annotations are actors' personal and collective memories in a CEHL and are in fact, a fast and effective way of learning in these environments. The annotations are very important as they provide us with a way for taking into account, in addition to the content of the

learning object, its uses, its assessments, and interactions between learners and teachers. Both structural and qualitative analysis of content, give the learning object an added value for different actors, as it allows to highlight the importance of the knowledge included into learning objects and those annotations attached [1].

In the field of e-learning, annotations are posted for a specific educational goal [2] as a trace of the activity of the reader, noticeable on a document as a mark placed in a specific purpose, and a specific location in which it cannot be dissociated. They can therefore contextualize the knowledge gained from learning objects. The actors then add gains in knowledge resulting from a learning process. Indeed, the annotations added to the learning objects involved in the readability of knowledge are not predictable [2]. Thus, sharing and reusing annotations allow achieving economic, computational, and educational opportunities as they are a real help in the drafting process, a reading support, and a content user evaluation [3]. A memory of collective annotations for all actors in a CEHL is son indispensable in order to capitalize and reuse learning's annotations. The CEHL actors benefit by sharing and reusing annotations. As a direct result of this annotation's knowledge capitalization, among others, the quality of learning will hugely improve [2], [4] and [5].

As part of this research work, we couple the Knowledge Management's concepts (as knowledge and collective memories) to the specific area of e-learning. Indeed, teachers, co-authors or tutors, for their teaching activities or learners during their activities of learning in a CEHL, manipulate a large number of learning objects supporting their activities. To memorize the items that they want to remember, these actors create different types of annotations on the objects to reuse as a working memory. Each actor constitutes an external memory for his own learning. In addition, [6] argues that KM systems and e-learning serve the same objectives: to facilitate the development of skills and learning in organizations. They are complementary. The e-learning systems are used to support learners so that they can develop their skills. They offer structured educational content and facilitate intercommunication on specific topics.

In our research, we focus on proposing an external memory, composed of all learning objects and their related annotations. This memory allows the teacher, for example, to store an idea, to find it quickly, or create an annotation in a given context with a view to reuse in another context. To be useful and usable for all actors in a CEHL, the memory must be well structured according to semantic annotations used and adapted to the current context of the activity of the actor. Thus, it will allow each actor to fluidly exploit annotations according to his cur- 
rent context. As a result, we will be able to offer an adapted learning to each actor according to his pedagogical objective and current learning context. This memory must be adaptable to different actors' learning activities according to their learning environment. In fact, we aim to propose an approach which will be then a context-aware memory of annotation for the capitalization and the reuse of learning annotations.

The adaptation of an application context can take many aspects, such us, behavior, content or presentation adaptation. In our approach, we focus on the adaptation of annotation memory to different learning contexts of different actors. Our objective is to provide these actors with learning adapted to their needs in different learning contexts. In addition, it is possible to reuse these different contexts, subsequently, in other contexts, and by the same actors or others. As a result, our approach will greatly increases the quality of learning by the annotations' added-value. It seems therefore necessary to propose a top level ontology of context. This ontology will consider, on the one hand, all the contextual specificities of all the actors in the annotation memory and ensures the sharing of different learning contexts, on the other hand.

To realize all the cited objectives, we propose OARLCAM (Ontology-based Approach for Reusing and Learning through Context-aware Annotations Memory). It is a new general architecture of the adaptive annotations memory with a detailed description of the functionalities offered by each module. OARLCAM is based on a context and annotation top level ontologies. The former represents the learning context of the various actors of the annotations memory. The latter describes the semantics of the annotation to be reused, shared, and learned from knowledge included in these annotations according to a pedagogical objective. The architecture of our annotations memory contains many interconnected modules. This facilitates its integration with other tools used by the actors of CEHL, for example, the e-learning platforms and annotations tools.

This paper describes a conceptual model and an ontology-based approach for reusing and learning through context-aware annotations memory. This memory manages annotations and adapts actors' (learner, tutor, teacher, and coauthor) behaviors to the various contexts of their activities. It offers a great reuse to share and have a better quality of learning. The annotation model that we propose is composed of three facets: cognitive, semantic and contextual. The paper is organized as follows. In the first section, we describe the state of the art related to our field of research, in the second, we present our approach, in the third, we devote to a context's top level ontology. In the fourth one, we present the top level ontology of the pedagogical objectives.

\section{STATE OF THE ART}

In this section, we present the basic concepts in our field of research then we present our approach of reuse and learning throw an annotation memory.

\section{A. Basic concepts}

Our research field of interest is located in the e-learning one. We are interested in providing learning through context-aware and annotations memory. Several concepts are involved inthis area namely, annotations, context, context- awareness and annotations memories. In the following sub-sections, we briefly introduce these concepts.

1) Annotation: The state of the art relieves several definitions of the Annotation concept [2] [4]. The most suitable to our work was given by [7], who considers an annotation as being "a trace of the activity of the reader, perceptible on a document as a mark, placed with a specific aim, and in a specific place of which it cannot be dissociated". This definition assumes that the annotation object belongs to the document. So, an annotation must have a well defined objective, based on the fact that the annotator did not annotate for nothing, but for a given goal. According to this definition, an annotation is regarded at the same time as an object and an activity. According to our readings, we define an annotation in these terms: An annotation is a particular note attached to a target. The target can be a collection of learning objects, an object, a fragment of object, etc.). An annotation has content, materialized by an inscription, which is a trace of the mental representation that the annotator is making to the target. The contents of the annotation could be interpreted by another reader (original author, tutor, learner, etc.). We call the anchor what binds the annotation to the target.

2) Context and context-awareness: According to Howe, the free on-line dictionary [8], a context is all that surrounds and gives a meaning to another thing. In this case, a definition of the context cannot be given in an isolated way without taking into account all the elements concerned with the context. This definition shows that the context must be external to the element concerned with this context.

In the field of ubiquitous computing (pervasive computing) [9] defines the context as any information that can be used to characterize the situation of an entity. The entity can be a person, a place, an object that is considered relevant to the interaction between user and application, including the user and the application itself.

A formal definition for the context is given by [4] as follows:

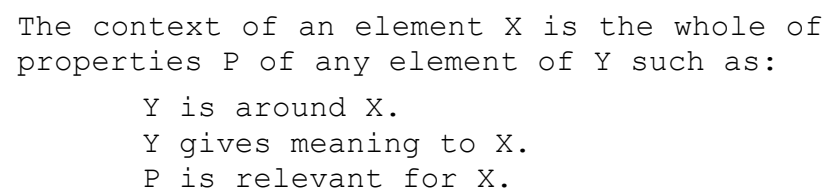

According to Chen [10], there are two types of context. The first is the active use of the context (automatically adapt the application behavior depending on the state of the context) and the second is the passive use of context (see the status of the context for the user or save this report for its future use).

In the literature, we find several definitions of contextawareness (conscience of the context). Schilit and Theimer [11] define the conscience of the context as being all the applications adapted to the context. Another definition given by Dey [9] specified that a system is considered as context-awareness, if it uses the context to give relevant information or a service to a user, knowing that the relevance depends on the activity of the user. In addition, context-awareness emerged in the fields of mobile and pervasive computing as a technique to design applications with a conscience of the environment, to ensure high level autonomy and flexibility. The context-awareness or the conscience of the context is known under other synonyms like adaptive or reactivate [12]. 


\section{B. $\quad$ Related Work}

Different models for describing annotations as activities where proposed, like [2], [4], [13], [14] and [15]. We describe the most recent and those specific to the field of learning.

During the last years, several researches were carried out to delimit the needs of CEHL actors and to extract the main elements helping to develop e-learning systems better adapted to their trainings and their needs. Although they are diversified, those researches do not take into account the capitalization of the learning experiments which can be exploited later by other actors. In fact, we think that the major challenge of e-learning nowadays is to integrate an approach to reuse the learning annotations within a CEHL.

1) Adaptation Approaches and personalized eLearning systems: In the last decades, researches were oriented to the adaptation and the personalization of the interfaces, in particular in the CEHL field. Indeed, several researches support the personalization in this field to guarantee a better satisfaction of learning.

One approach consists to allow teachers to make scenarios for all the learner's uses possible of the system [Lejeune]. A teaching scenario describes goals and learning situations while defining how the learning objects will be implemented in a precise context of training. However, the teaching scenarios help teachers to integrate the CEHL into their work practices, but do not allow creating sequences of activities adapted to each learner's competences.

A second approach, [16] and [17] for example, devotes a part of the e-learning system to be personalized by the teacher. Thus, teachers can parameterize the generation of the activities or select the activities which are appropriate for their learner. This personalization is done manually by the teacher, without bond with possible learner profiles, and can't be considered as based on a generic or unified model. In fact, each teacher can use several e-learning systems and must control several environments of personalization to succeed in defining in each case his teaching choices. Moreover, the teacher needs to profit from its experiments and those of the other teachers, in addition to the tutor's ones. We notice that this second approach is only interested in the training of learners and is not based on models.

A third approach [18] consists in personalizing the elearning systems automatically so that their contents are adapted to each learner's knowledge. This personalization can progressively be made throw the learners' answers (as a result to their behaviors) and uses the stereotypes associated to the learners or the learner model according to the e-learning systems. This automatic personalization is adapted to the system's knowledge about learner but is not always adapted to the teacher's learning goals.

Each one of these approaches answers only to a part of the problem, but does not provide a solution to the whole: the adaptation of CEHL to the activities of the teachers and the adaptation of their contents to each one of their learners [19].

We present, in the following, some examples of projects working on the adaptation and the personalization in the e-learning systems.
2) Approaches of annotations memories in $e$ learning: The recent work on the annotations memories, [Ouadah] proposes a context-aware annotation tool for an external annotations memory for the teacher. It is based on two architectures to propose their adaptive annotations tool general architecture. This tool is able to identify the teacher's activity current context in order to adapt itself to the teacher's behavior and his activities' changes.

This approach supports the teacher's annotation activity to reuse it in another context. However, it is not an annotations memory for a general learning. Indeed, it can be integrated into an annotation tool and not into a CEHL. In addition, it is dedicated to only one actor who is the teacher.

As a conclusion, we can deduce the lack, in the literature, of an adaptable approach for the training of all CEHL actors based on annotations according to a given context.

\section{OVERVIEW OF OUR APPROACH}

In order to take into account all the mentioned lacks in the last section, our annotations memory includes three subsystems: i) the contextualisation subsystem, containing the modules of context capture, context handling, context server, context presentation and a context top level ontology of training, ii) the learning subsystem, containing the modules of learning objects management, learning objects composer and follow-up of the training and iii) the annotation subsystem, containing the annotation module, the annotations' manager, the annotations' adapter, the annotation top level ontology, the annotations' presentation module and the annotations warehouse, for later reuses. The following sections describe all these subsystems' modules.

\section{A. Context management}

This subsystem determines the aspects of our contextawareness approach.

1) The context capture module: is carried out using a whole of services which interact with different elements of $a$ given context (operating system, learning objects manager, organizer, etc.). This interaction is made in a direct way; when the context information is accessible, or in an indirect way, thanks to a context export operation from the context source to this module.

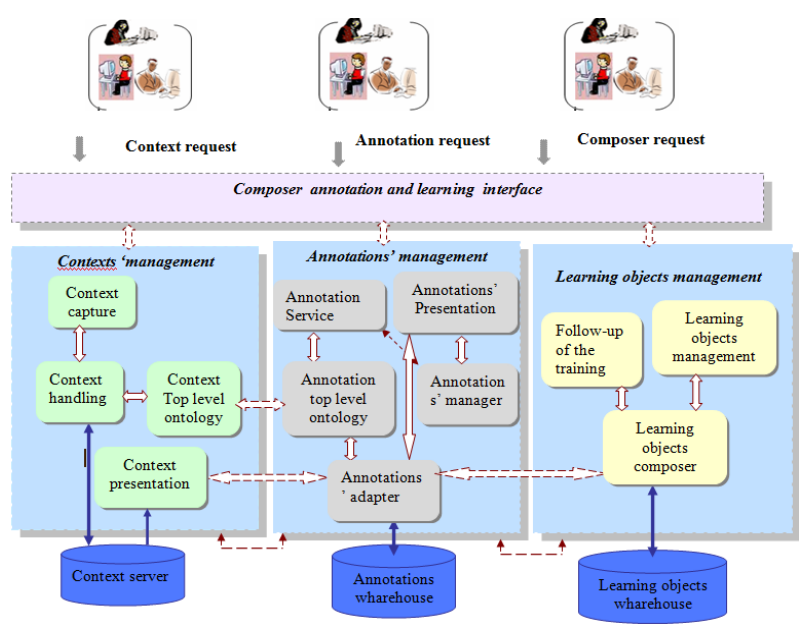

Figure 1. Our approach OARLCAM 
2) The context handling module: Context's information, provided by the context capture module, are treated according to our context top level ontology in order to be stored in the context server. This treatment consists in making a mapping between data types of the context source and our context model.

3) The context server module: The context data are stored in XML format in order to facilitate their sharing and their use by adaptive applications and to keep the contexts history.

4) The context presentation module: context information is presented by this module. It can be consumed by other applications to be adapted to their context. This module is a service which gives information about the current context to the annotations' adapter module (the details of this presentation are not in this paper), knowing that those information are extracted from the context server.

\section{B. Annotations management}

The main objective of the Annotation management subsystem is to allow the Sharing and the reusing of annotations. To do so, this subsystem is composed by the following modules.

1) The annotation module: allows actors to add an annotation according to our annotation top level ontology

2) The annotations' manager module: manages the annotations affixed according to our annotation tool, for example, to add, to modify or to remove an annotation.

3) The adapter annotations' module: adapts the annotations stored in the annotations warehouse according to the training context (objective) provided by the context presentation service. This service also provides the adaptation to the services of learning objects composer to combine them with these objects (annotations source) or to provide them directly to the annotations' presentation module (the result of the request). For example, one learner during his revision wants to extract from a learning object all the related explanatory annotations (in an explanatory context). He also wants that this annotation will be posted only during its next envisaged revision in a given day and a given hour. In this case, the service of annotation checks the properties of the context provided by the context services and posts the annotation only if the context is verified.

4) The annotation top level ontology module: is a generic and exhaustive annotations ontology which provides the semantics of different learning annotations. We develop this ontology to mitigate the various insufficiencies in the state of the art.

5) The annotations' presentation module: presents, for the different architecture actors, the annotations adapted to the learning context. These annotations are treated to be adequate to the actors' requests and their learning objectives.

\section{Subsystem of the learning objects management}

The subsystem of learning objects management is composed of:

1) The learning objects management module: is used to handle the learning objects of our approach. It allows actors to create, to add, to remove, and to modify learning objects.
2) The learning objects composer module: serves to compose the learning objects with existing annotations for a given learning objective and a given context. The learning object can be composed with the explanatory, descriptive, critical or prescriptive annotations.

3) The follow-up of the training module: serves to save the learning activities history of our memory's actors.

\section{The Top Level Ontology CONTEXT (TLCO)}

TLCO is a generic and exhaustive context ontology which provides the context proprieties related to learning provided by our annotations memory. It is conceived to solve the limits and the insufficiencies of the existing context models.

For a given learning objective and a given context, we must extract the adequate annotations from the annotations memory. We use then a mapping method for determining the similarities between a learning context (context top level ontology) and annotation semantics (annotation top level ontology). The annotation top level ontology contains three facets: cognitive, semantic and contextual. On another side, the context top level ontology contains six facets. Four of these facets (user, activity, environment, collaboration) where defined by [5]. We added two more facets (composition and objective) to have an exhaustive context model which takes into account the context of reuse of learning and the learning objective. The context use is very important for applications that frequently change dynamically their environment. Context is all information that characterizes a situation of a person or an object and describes everything surrounding the considered object and gives it meaning.

Several models are used to formalize the context information. The choice of a particular one depends on the needs and characteristics of the model itself. However, most existing methods fail to propose a generic ontology to model context [20], in particular, they do not take into account all the actors of an annotations memory.

In addition, our top level context ontology is essential in our research about a new architecture for learning through context-aware annotations memory. This architecture [21] is based on the context top level ontology to represent the learning context for the different actors of the annotations memory and a generic ontology describing the semantics of the annotation for future use, share and learn from the knowledge it included in a given learning objective.

In the first section, we demonstrate the need of context in a learning based on annotations. We, then, present a state of the art of context models in both mobile and elearning applications. Thus, we deduce the importance of having a context's top level ontology developed in the third section.

\section{A. Motivation of context in a learning based on annotations}

Learning through context-aware annotations memory is automatically adapted to different learning contexts. In this kind of memory, adaptation depends on a set of parameters, such as: the name of the annotator, place, time, etc. It is therefore important to identify what are the relevant data that form the precise learning context. The recognition of the learning context is important in order to 
adapt the training according to the context and the learning objective. All these parameters and others can form a particular learning context based on learning annotation, so that users access to the memory and the knowledge included in the annotations specifying parameters which formed the learning context based on annotations. In order to model the learning context, we use the concept of ontology, which provides an explicit specification of a conceptualization [Gruber 93]. Our context top level ontology will then allow a unified, coherent, shared and reused learning in different contexts.

\section{B. TLCO modeling approach}

There are several methods for ontology development (top-down, bottom-up and combined). To design our context ontology of annotations learning, we follow the iterative method proposed by Noy [22]. We describe below each step of this method.

The field and scope of the ontology: We begin the ontology development by defining its domain and scope, answering the following questions: What is the domain that the ontology will cover? What are the goals of ontology development? and Who will use the ontology?

Our ontology field is the learning context based annotations of all actors. Our context ontology includes therefore concepts related to the learning activity of the learner, the teacher's teaching, the tutor's tutoring and the co-authors collaboration. This ontology is designed to integrate both the active and passive context (the learning context) as part of learning annotations memories. Moreover, our ontology is not intended to be used by a human, but by the different components of context management to provide learning-based annotations in a given context.

1) The field and scope of the ontology: We begin the ontology development by defining its domain and scope, answering the following questions: What is the domain that the ontology will cover? What are the goals of ontology development? and Who will use the ontology?

Our ontology field is the learning context based annotations of all actors. Our context ontology includes therefore concepts related to the learning activity of the learner, the teacher's teaching, the tutor's tutoring and the co-authors collaboration. This ontology is designed to integrate both the active and passive context (the learning context) as part of learning annotations memories. Moreover, our ontology is not intended to be used by a human, but by the different components of context management to provide learning-based annotations in a given context.

2) State of the art of Context ontology: In the literature, we find several context ontologies. Some concern the context of mobile applications, and others are dedicated directly to the context of e-learning applications. We present in the following relevant properties of the user activity context in mobile applications and e-learning applications classified in groups.

a) Ontologies in mobile applications: based on the works presented in [Miraoui],[ferry],[Aubry] and [chaari], context ontologies in mobile applications can be summarized in the four following categories: i) The user context (User profile, its role, its activity, etc.), ii) The environmental context (such as brightness, noise, temperature), iii) The machine context (these are the properties of the computer system as the operating system) and iv)The spatio-temporal context (location, time). b) Context ontologies in e-learning applications: we notice that there are few ontologies which are related directly to the context in the e-learning applications. These context ontologies are according with those proposed in mobile applications in addition to specific e-learning concepts such as, pedagogical resources and activities [Grandbastien], organizational role [6], [23] and collaboration [4] or [5] presents the context ontology in four partially facets: i) User Context (allows access to information according to user identity), ii) activities Context (allows the presentation of appropriate learning resources to help the user to accomplish his task, configure HumanMachine interaction depending on the complexity of the task (free hand, hand busy, etc.), iii) Environment Context (allows to locate the site, object recognition, mobility management, etc.) and iv) Collaboration Context (contextualizes other users whom can potentially work, configure the forms of collaboration (message, voice, video, etc)).

In conclusion, the different facets of the context ontology presented in this state of the art, are not specific to all learning contexts of different annotations memory actors (teachers, learners, tutors and co-authors) and did not explicit the aspect of "pedagogy" of the pedagogical activities of these actors. In addition, these context ontologies did not take into account for subsequent reuse of different learning situations in future contexts. These reuses are fundamental to the sharing of knowledge and learning experience feedback, thus increasing the learning quality. As a consequence, the top level context ontology is essential to cover the learning context for all actors of annotations memory.

The following section describes the characteristics of our top level context ontology, deduced from the state of the art of mobile (pervasive) and e-learning applications.

\section{3) Deduction approach of the ontology}

To identify the characteristics of our top level context ontology, we apply the context activity definition presented above. We identify the relevant properties of the elements / concepts that are around the learning activity of the different actors of annotations memory and that are relevant to them. These concepts are essential to learning through annotations memory. We apply the following steps to derive our ontology:

a) Step1: Use the concepts and properties provided by mobile applications' ontologies:

For the passive use of the context, we keep the user profile (A user is an actor of a memory annotations)

For the active use of the context, we keep the computational environment (hardware and software) and user profile (A user is any actor of a memory annotations) as our system adapts the content based on these two elements.

The physical context is irrelevant to the learning activity.

b) Step2: Use the concepts and properties provided by e-learning applications' ontologies

For the passive context, we maintain the learner activity, the teaching activity, the learning domain and place. These concepts are relevant to memorize learning-based annotations. We add the tutoring and collaborative activities.

For the active context, we keep the learning activity, the teaching activity and learning domain, as our system 
adapts its functionality according to these elements. For example, it will filter the annotations according to learning activity. We add the tutoring and collaborative activities.

c) Step3: Complete the top level ontology with the relevant concepts missing

We add missing and primordial concepts to existing facets which are indispensable for learning based on annotations. For example, the tutoring and collaborative activities at the activity facet, etc.

We add two more facets describing the learning reuse context and the learning objective context

Thus, we structure the top level context ontology with a top-down approach starting from the most generic concepts and we follow an iterative process, where we enhance the ontology concepts and their structure, in each iteration.

\section{Top Level Context Ontology (TLCO)}

The Top Level Context Ontology (TLCO) allows our annotation memory to have a current representation of the context object [ 24]t. With this ontology, our memory becomes conscious of the current context and can therefore adapt its services to different actors according to changes in their environment.

TLCO represents the properties of the current context to provide their values to our annotation memory. It uses these values to specify the contextual aspect of an annotation in an active learning situation, but also to adapt the content according to the current learning context. Based on the various proposals of the state of the art, we propose the TLCO (see fig 1), whose attributes are organized into six facets, specifying mainly the new dimensions and concepts proposed.

1) The Activity Contex facet: is determined by five elements namely the learning domain, the learning activity, the teaching activity, the tutoring activity and the collaborative activity. ing

a) Learning domain: is the knowledge domain dur-

b) the learning based annotations memory (databases, programming...).

c) Learning activity: allows the presentation of appropriate learning resources to help the user to accomplish his task, configure Human-Machine interaction depending on the complexity of the task (free hand, hand busy, etc...), Tracing the tasks' progress, etc.

d) Teaching activity: corresponds to the teaching activity carried on by the teacher (course preparation, correction, explanation of a concept...).

e) Tutoring activity: concerns the tutoring activities performed by the tutor (add a course, extend exercises, reexplain a concept .....).

f) Collaborative activity: represents the activity of collaboration between course teachers (documents sharing, reorganize course, add a reminder .......).

2) The Environment context: is described by two components which are the date and place:

a) Date: describes the exact moment of learningbased annotations, it includes

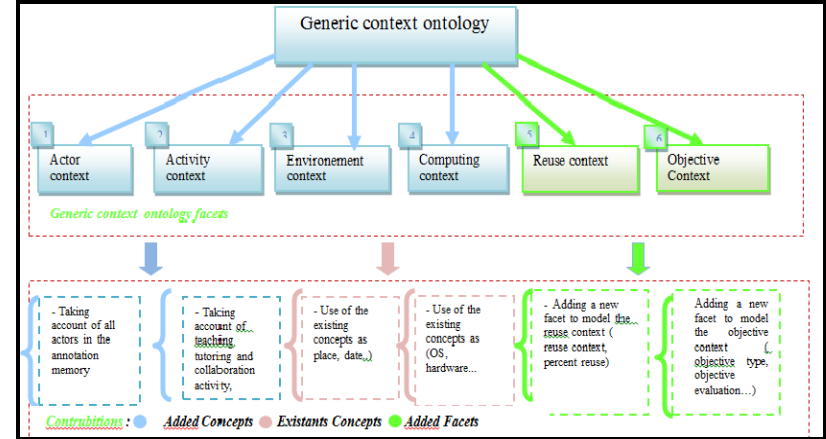

Figure 2. TLCO facets

b) Location: is the location of the actor (learner, teacher, tutor and co-authors) when using the learning environment based annotations.

3) The Computing context: specifies the equipment and tools used by the actor during his learning activity, such as:

a) Operating System: means the operating system installed on the host machine (Windows, Linux, Symbian OS X ...).

b) E-learning platform: means the e-learning platform used by the actor, it can change from one context to another if the actor handles more than one e-learning platform Organizer.

c) Organizer: is used by different actors to organize and plan their intervention programs on the learning environment.

d) Hardware: describes the machine on which the learning environment based annotations is running.

4) The Context reuse is described by two components namely the reuse identifier, determining each annotations' use, and reuse percent, rising the importance and quality of learning through these annotations:

a) ID reuse: each reuse is identified by its number of use.

b) Reuse Percent: indicates the frequency reuse of current learning context.

5) The Learning objective context: specifies the learning semantics through the annotation memory. It consists of three components to know the learning objective, the objective type and learning evaluation.

a) Learning objective: is the pedagogical objective of each actor as an active learning situation. The objective differs according to each actor. The objective explicit the semantics and utility of the actor's activities learning witch used for several subsequent reuse by the same or different actors in the annotation memory.

b) Objective Type: indicates the level learning of the actor (average, expert, beginner or advanced).

c) Learning evaluation: consists of all possible evaluations that are available for a learning environment. It includes the evaluation of the actor and the content.

The following figure represents the conceptual model of TLCO.

The figure 3 shows TLCO classes hierarchy using the editor protégé. TLCO will be achieved through the integration of different facets.

The following paragraph describes the Top Level Pedagogical Objective Ontology (TLPOO) . 


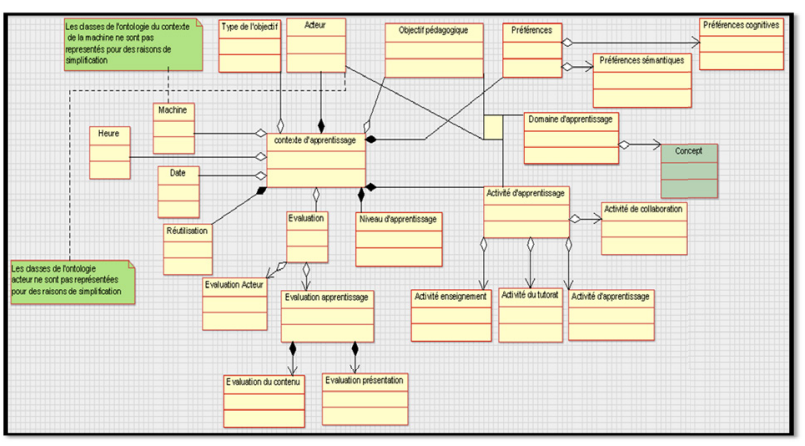

Figure 3. TLCO Classes Hierarchy

\section{Top LeVel Pedagogical Objective Ontology} (TLPOO)

The Top Level Pedagogical Objective Ontology is defined to describe the learning objectives for the contextaware annotations memory, in which learning annotations are capitalized and reused for learning-based annotations.

The state of the art of learning objectives ontology reveals the lack of an objective ontology dedicated to all the actors in a CEHL. Indeed, a TLPOO is essential to our context-aware annotation memory. It allows covering all actors' pedagogical objectives of the learning activities. In addition, annotations were created initially for one purpose and are capitalized and reused in our context-aware annotations memory for the same purpose for which they were created or for other purposes. Thus, pedagogical objectives are closely correlated with the semantic of learning annotation.

In fact, by explaining these pedagogical objectives, we are expounding the semantic aspect of the annotation object [2] [4] [5]. This semantics is essential to extract the appropriate annotations in a given learning context. Thus, the learning objectives related to the semantics of the annotation are explicated as ontology dedicated for the learner [mile] or the teacher [ouadah] and not both.

Our approach to reuse annotations and learning contexts is based on a mapping between the TLCO [24] and the TLPOO [25] to extract the appropriate annotations to a given learning objective. This mapping is in fact a correspondence between the pedagogical objective of an actor in a given learning activity and the pedagogical objectives of the annotation semantics. Then it seems to us, necessary to propose a top level learning objectives ontology [25]. This ontology will take into account, on the one hand, the pedagogical objectives of all the actors in the annotations memory and will ensure the learning and the reuse of annotations memory, on the other hand. Thus, this TLPOO allows providing the appropriate annotations for a given pedagogical objective.

\section{A. TLPOO modeling approach}

To design the TLPOO, we follow as fit TLCO the Noy [22] iterative method. We describe below each step of this method.

1) Step 1: Field and scope of the ontology: We begin the ontology modeling by defining its domain and scope and this by answering the following questions: What is the domain that the ontology will cover? What are the goals of ontology development? And Who will use the ontology?

The field of our ontology is the learning objective explaining the learning semantics of annotation for all actors, in addition to the learning objectives of the learning active context. Our TLPOO therefore includes all concepts describing the learning objectives of annotations and learning contexts for all the actors of the annotations memory. For this, it is a generic and exhaustive pedagogical objective ontology.

The ontology is designed in order to formalize and clarify the semantics of annotations produced by actors (annotation objective) and the semantics of a given learning context (pedagogical objective). This formalization allows us to reuse and capitalize annotations in a context-aware annotation memory. Moreover, TLPOO will be explored by the different context components and the annotation management providing context-aware learning-based annotations.

In the following section, we will briefly present some the most important objective ontologies in the literature.

2) Step2: Reuse of existing ontologies: The objective of this step is to reuse existing ontologies even if they have a different objective from TLPOO. We can reuse again some or all of these ontologies after we adapt them to our needs. The most recent researches about pedagogical objective are those of [2] [13][14][15]. Mille, for example, proposes in [4] a formalization of ontology of annotation objective dedicated to the learner. This ontology contains generic concepts for annotation purposes. However, these objectives are not specific to the teacher, the tutor nor the co-

a) authors and do not clarify the aspect of pedagogy of learning activities of these actors. Mokadem [13] also proposed a pedagogical objective ontology dedicated only to the learner.

In order to cover all the aspects in our TLPOO, we consider some ontologies despite they are not specific to the annotation objective of different actors. It is the case of LOM, which identifies the concepts describing learning resources and IMS-LD, which identifies the necessary concepts for modeling a learning process.

3) Step 3: Identification and organization of the ontology concepts: In addition to the four ontologies mentioned above, we enrich TLPOO taking into account the specific learning activities to all actors which are the teaching (teacher), learning (learner), tutoring (tutor) and collaboration (co-authors). To do so, we used the concepts and properties provided by learning ontologies, [LOM] and [LMS ID] and those of pedagogical objective ontology of annotation, [mile], [desmoulins] and [mokadem]. We than, complete TLPOO with the relevant concepts missing and primordial ones as they are indispensable for learning based on annotations (the tutoring activity, collaborative activity at the activity facet).

In this design process, we adopt a top-down approach starting from the most generic concepts and we follow an iterative process, where we improve the ontology concepts and their structure in each iteration.

The figure 4 presents the ontology concepts structured by the "is-a" relationship. 
TABLE I. TLPOO CONCEPTS

\begin{tabular}{|c|c|}
\hline 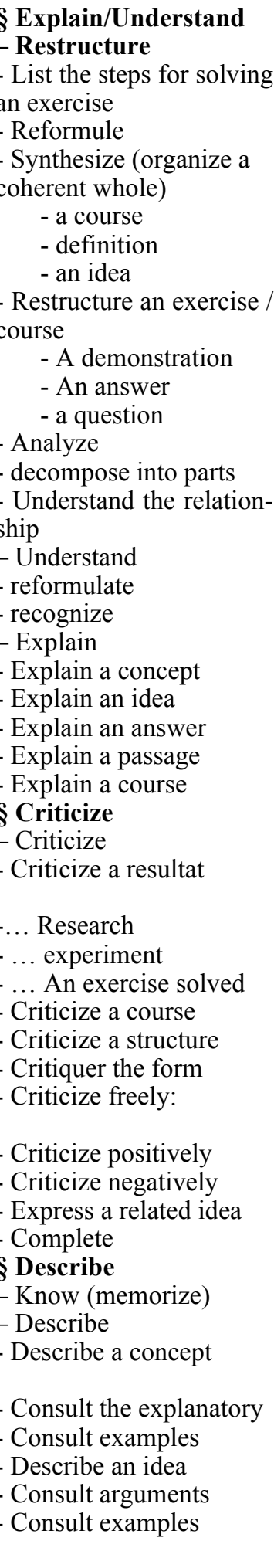 & 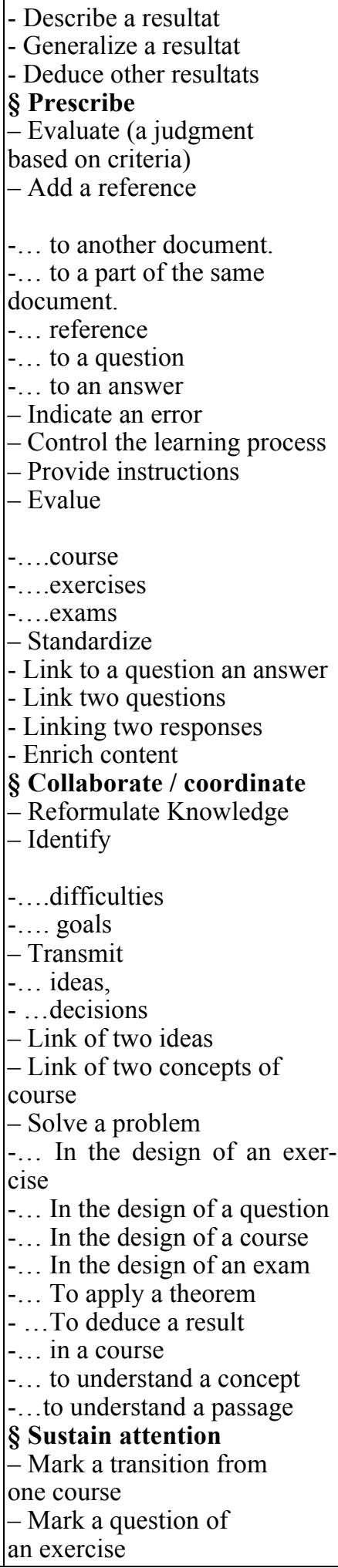 \\
\hline
\end{tabular}

\section{B. Ontological modeling of an exhaustive "learning context"}

Ontological modeling of a learning context is considered as an important step in our work. The purpose of this step is to identify all the characteristics of an exhaustive learning context instance of a learner, teacher, tutor and other co-authors on the one hand, and to clarify the se-

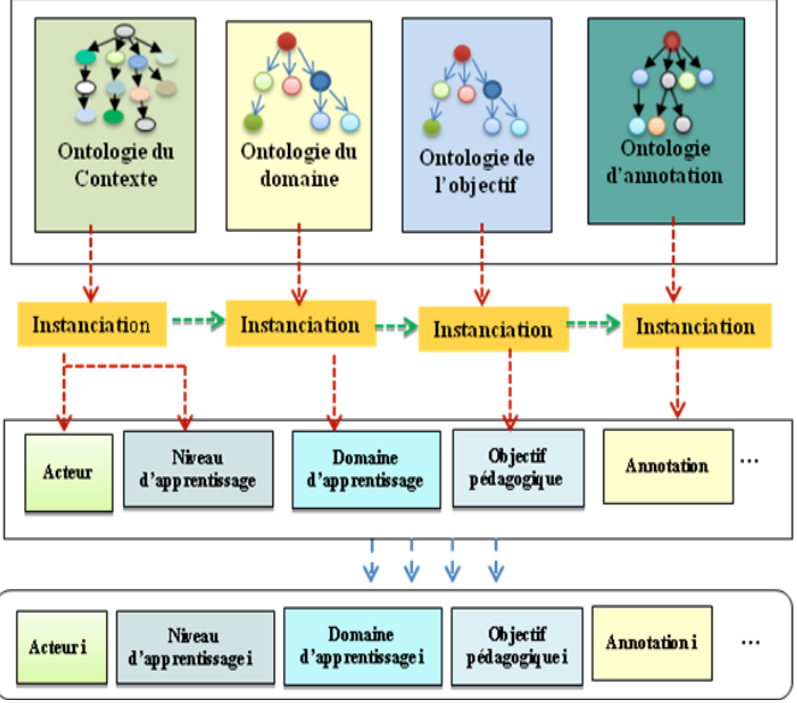

Figure 4. Ontological modeling of a complete learning context in stance

mantic relationships that may exist with different ontologies of the annotations memory. This step prepares the integration of ontological reasoning namely the alignment between ontologies contributing to a complete instance of a learning context (Cf. Figure 4) (ontology annotation, ontology, ontology of context, ontology of the target).

In the vision of knowledge reuse, the first two attributes of the learning context for example (actor and level of learning) will be obtained based on the concepts described in the sub-ontology of the actor and that of the learning field. This is in order to develop a larger ontology as illustrated in the previous figure called complete instance learning context. That is to say, the learning context increased adequate annotations after their adaptation according to learning objective and learning activity of each actor. Thus, we explicit in the next section our generic learning objective.

\section{An ontology-based approach for learning annotations and context reuse}

We expose our approach and process for reuse of annotations and associated learning contexts through describing the mainly steps defined. This is to provide to the actor, an appropriate learning which is reviewed or validated previously by others, with similar learning contexts. The modeling and the formalization of learning annotations and learning contexts will allow defining functions for their comparison and their evaluations, in order to reuse them. We propose our method for measuring similarities in order to provide learning annotations dedicated to a given and well-defined pedagogical goal. An alignment between the two ontologies (Annotation and the context ones) will provide us with a learning based on annotations according to the current context which requires a pertinent criterion of similarity between the learning contexts and the learning annotations.

Accordingly, we then present the key steps for reusing annotations and contexts learning. We combine ontological engineering (Modeling, alignment, similarity measurement...) and context-awareness techniques in our approach to reuse. 
Our system detects the learning context proprieties (Learning objective, type of activity, level of learning, course or pedagogical object...) of actors and starts to search for a similar learning context in the context server. If it finds a similar learning context, it is added to the current contextual data (Date, location, hour...) then presents the relevant learning annotations to the actors. Once the learning session is over, the actor shall have the ability to express his level of satisfaction for the learning provided by our system. (Satisfied, unsatisfied, fairly satisfied, well satisfied)[aloui].

The following section presents our process PRALC. Then, we describe our method of measuring similarities on which is based our approach for reuse.

\section{APPROACH FOR THE REUSE OF LEARNING ANNOTATIONS BASED ON ONTOLOGIES ALIGNMENT}

Our approach of learning annotation reuse lies essentially on a similarity between the two ontologies of context and annotation respectively to the subsystems of context and annotation. This has for objective to check the annotations which are adequate to the current learning context taking into consideration all the contextual properties which describe it. Our approach of reuse is concerned with the levels of reuse 1) the reuse of stored annotations to take advantage of the included knowledge, benefit from experiences feedback of different actors and consequently, improve the learning quality always according to a pedagogical objective and a current context; 2) the reuse of learning contexts stored in the context server within similar learning contexts by other actors 3 ) the reuse of the same learning context by the same actor through the planning of further users.

\section{A. ARLAOA Description}

Our approach of reuse can be described by a four-step cycle namely (Cf. Figure 5): the search for similar con-

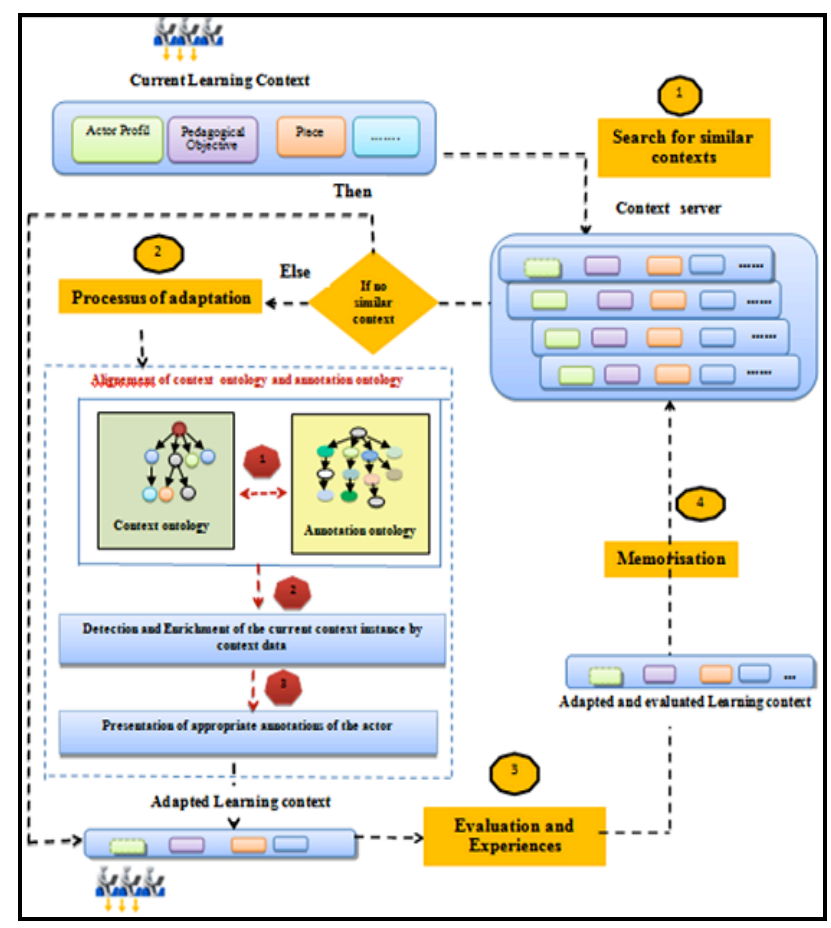

Figure 5. ARLAOA Approach texts, the process of adaptation, the memorization and the evaluation (experience feedback). An actor shall start by defining his request; He shall so, define his pedagogical goal, his pedagogical activity which corresponds to the goal facet and that of the activity-tasks of our context ontology [26]. Our system will apply the approach of reuse in order to provide the actor with the suitable learning adapted to his learning context.

Our reuse approach for an annotation memory is thus summarized in the following four steps.

\section{1) Step 1: Searching a similar context}

To meet the actor's needs for a learning adapted to his learning context X, we will start by searching, in the context server, for a learning context similar to $X$. If it is the case, we will enrich this context instance with the current computing contextual properties such as the date, the location, etc., and then we present it directly to our actor. At the end of the session, the actor shall evaluate the provided learning. Our system memorizes this learning context in the context server (implementation of the useful data). If we don't find from the beginning a similar learning context, we will go to the adaptation process in order to extract out a learning adapted to our actor.

\section{2) Step 2: Adaptation}

This step is primordial for adapting the learning annotations to the current context through the alignment of two ontologies of annotation and context. This alignment shall allow checking the annotations which correspond to the same properties of the current learning context (pedagogical goal, pedagogical activity...). We will adopt then an approach of measurement of semantic and structural similarities which are expressed by a better learning quality. Owing to this alignment, the checked out annotations will be enriched by the contextual data detected by the system context capture. Indeed, it is a learning based on contextaware annotation memory.

\section{3) Step 3: Memorization}

Once we provided the actor with a learning adapted to his learning context as well as to his pedagogical goal, the learning-context instance is stored in the context server for further use within similar contexts by other actors or by the same actor.

\section{4) Step 4: Evaluation and Experience Feedback}

The result/evaluation characteristic defines the level of pertinence of a learning session by our system. It is a criterion which shall be taken into account during the stage of reuse of previous learning contexts. In fact, the reuse lies, on one hand, on the learner's judgments concerning the pertinence of the quality of learning proposed also by other actors such as the time span which separates successive uses. During his learning, our actor then evaluates the pertinence of his current learning context.

Our reuse approach is actually based on interactions between different ontologies making explicit learning by context-aware annotation memory. The figure 6 illustrates this interaction.

In consequence, this approach lies on a similarity between the annotation ontology and the context ontology in order to check the adequate annotations for a given learning context. We will, then, use our own algorithm of similarity measurement which shall be presented in the following section. 


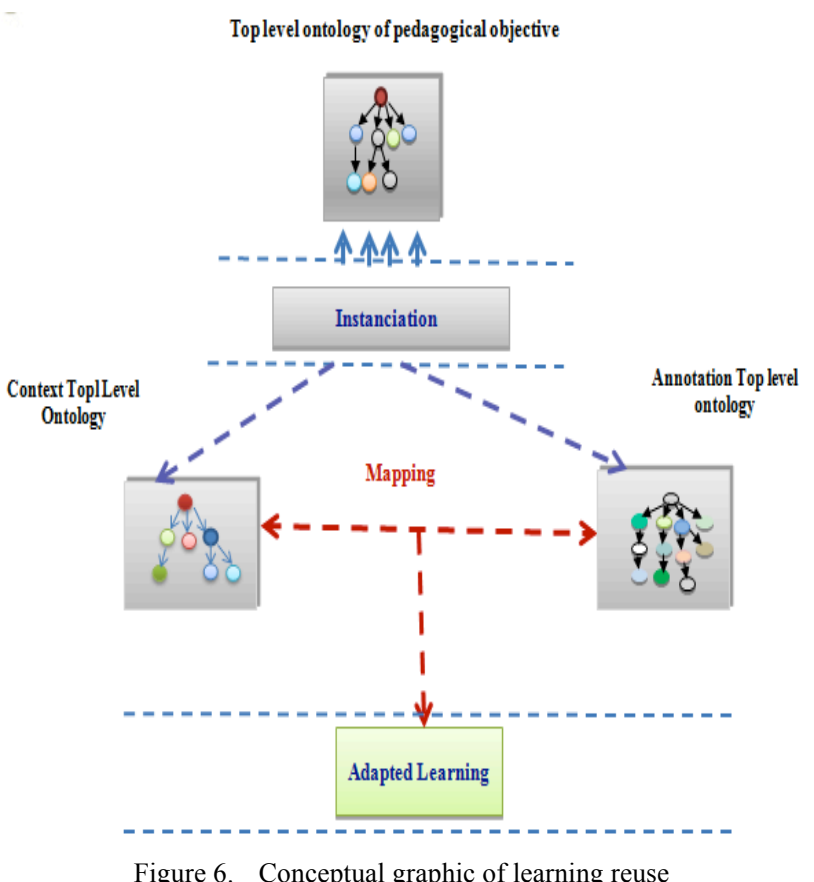

\section{B. Illustrative scenario}

When reviewing its course of databases, a learner $\mathrm{X}$, in fact, needs the remarks, comments, explanations, improvements. In general explanatory annotations, analytical, prescriptive are essential to an understanding of its course. In some cases, these different annotations can also result from a negotiation with the teacher to provide such additional exercises and satisfactory progress and learning.

The learner will then connect to our system for a precise pedagogical purpose: "Understand the database course". He expresses his request to the system in order to be provided with all the explanatory annotations related to the course. Our system detect the learning context proprieties (learning objective, type of activity, level of learning, course or pedagogical object...) of this learner and starts to search for a similar learning context in the context server. If it finds a similar learning context, it will be added to the current contextual data (date, location, hour...) then presents the relevant learning annotations to the learner. Once the learning session over, the learner shall have the ability to express his level of satisfaction for the learning provided by our system (Satisfied, Unsatisfied, Fairly satisfied, Well satisfied). This evaluation will help us later on to evaluate the learner's training and have an idea about the amount of achievement of our pedagogical objectives by the corresponding learner. This could be useful for us to reassess the most valid and satisfactory learning contexts in the system. The amount of achievement of a goal can be expressed in the form of a percentage which is defined by a tangible figure restrained between 0 and 1 .

Unless the system finds a learning context similar to the learner's current context, the adaptation process is triggered in order to extract the adequate annotations out of the annotations memory, an alignment is then performed between the context generic ontology and the annotation generic ontology to recover the annotations corresponding to the same instances of the current learning context.
Then, the system adds up the adequate annotations to the current contextual proprieties and presents the learning based of annotations to the learner.

Our system supports all the actors together at the same time (teacher, learner, tutor, and co-author) in order to represent, share and capitalize their knowledge included in the annotations (annotation memory) of the e-learning field. The capitalization of learning contexts will be exploited later on by other actors. In our approach, we rely on the techniques of alignment and similarity measurement for the adaptation of the learning annotations to a given learning context.

In the next section, we describe our method of similarity measurements between the context ontology and the annotations ontology, on one hand, and the various instances of context ontology for further reuses of the learning context on the other hand.

\section{Similarity measurements}

\section{1) Measure of semantic similarities}

We use the Jaro-Winkler functions as they give good results of similarity according to the comparative study of Cohne [27].

Moreover, recent works [albohassani] [Fellah] and [touzi], for example, have shown the usefulness of WordNet in identifying synonymy between concepts of a given domain. We expect that our future experiments our algorithms are based on Wordnet, in addition to the measurement function.

The SimSEM function allows calculating the semantic similarities of the pairs of concepts $\mathrm{C} 1$ and $\mathrm{C} 2$ respectively of both ontologies $\mathrm{O} 1$ and $\mathrm{O} 2$ by adopting the following steps:

For each concept $\mathrm{C} 1$ and $\mathrm{C} 2$, go through the concepts of $\mathrm{O} 2$ so that the category of $\mathrm{C} 1=\mathrm{C} 2$,

Calculate the semantic similarity according to the JaroWinkler [12] function,

Store the semantic similarity (SimSEM) in the vector VSS.

\section{2) Measure of Structural Similarities}

The structural techniques exploit the ontology structure to be compared, often represented in graphics. The comparison of similarities between two concepts of both ontologies can be based on the position of the concepts in their hierarchies. These techniques are based on the following hypothesis: " $(\mathrm{H})$ - if two entities of two ontologies are identical, their nearby entities will be the same in a certain way" [28] and [29].We suggest calculating the structural similarity between the entities of two ontologies. We are inspired from the works of Albohassaly [15] and Zghal [30]. We calculate then the structural similarity by exploiting the semantic similarity of the pair of concepts to match as well as the contiguous structure by adopting the following steps:

If the contiguity of a concept $\mathrm{c} 1$ noted $\mathrm{V}(\mathrm{c} 1)$ is similar to the contiguity of the concept c2 noted $\mathrm{V}(\mathrm{c} 2)$ then $\mathrm{c} 1$ and $\mathrm{c} 2$ are similar in a certain way,

The position of $\mathrm{V}(\mathrm{c} 1)$ in relation to $\mathrm{c} 1$ shall be similar to $\mathrm{V}(\mathrm{c} 2)$ of $\mathrm{c} 2$, then we suggest to calculate the structural similarity of the pair $(\mathrm{C} 1, \mathrm{~V} 1)$ and $(\mathrm{C} 2, \mathrm{~V} 2)$ so as to get Simst $(\mathrm{C} 1, \mathrm{~V} 1)$ in order to determine the position of $\mathrm{V}(\mathrm{c} 1)$ in relation to $\mathrm{C} 1$ and respectively $\mathrm{V}(\mathrm{c} 2)$ in relation to $\mathrm{C} 2$. 
Structural Similarity Algorithm Function: Sim ST INPUT:

1) $\mathrm{O} 1$ et $\mathrm{O} 2$ : the two ontologies to align

2) VSS: vector of semantic similarity

3) II S: Similarity Weight of each category of concept OUTPUTS: VSST: vector of structurel similarity BEGIN

/* go to each concept of ontology $1 * /$

For each $(\mathrm{CO} 1 \varepsilon \mathrm{O} 1)$ do

$/ *$ go to each concept of ontology $2 * /$

For each $(\mathrm{CO} 2 \varepsilon \mathrm{O} 2)$ do

If $\mathrm{C} 1$. type $==\mathrm{C} 2$.type then $/ *$ Extract in VV1 vector neighboring concepts to $\mathrm{C} 1 * /$

VV1 $=$ NeighboringCONCEPTS $(\mathrm{C} 1)$

/* Extract in VV2 vector neighboring concepts to $\mathrm{C} 2 *$ / $\mathrm{VV} 2=$ NeighboringCONCEPTS $(\mathrm{C} 2)$

/*Calculate the $\mathrm{C} 1$ structure of neighborhood similarity */ SimSV1 $=$ fonctSimSV1 $(\mathrm{C} 1, \mathrm{VV} 1)$

/* Calculate the $\mathrm{C} 2$ structure of neighborhood similarity */

$\mathrm{SimSV} 2=$ fonctSimSV2(C2,VV2)

/*calculate structural similarity*/

SimST $=$ fonctSimST $($ SimS, SimSV1, SimSV2,VV1,VV2)

/* Add $\mathrm{C} 1, \mathrm{C} 2$ and SimST to VSST*/ Add $((\mathrm{C} 1, \mathrm{C} 2$, SimST), VSST)

END Return (VSST)

Figure 7. Structural similarity algorithm

Several functions measuring structural similarities based neighborhood calculating are proposed in the literature [29], [31] [32]. The choice of a particular function depends closely on the results obtained. We are currently conducting a comprehensive study on the selection or improvement of any of these functions to suit our research. For the example in Figure 3, and to illustrate our algorithms, we use the measure of by ex-ploiting the similarity measure neighborhood Wu \& Palmer [17] (simWp) and the semantic similarity measure Jaro- Winkler (SimS) in the following manner:

$\operatorname{SimStr}(\mathrm{c} 1, \mathrm{c} 2)=\Sigma \operatorname{SimS}(\mathrm{VV} 1, \mathrm{VV} 2) * \operatorname{SimWp}(\mathrm{c} 1$, $\mathrm{c} 2) *\left(1-|\mathrm{d} 1-\mathrm{d} 2|^{2}\right)$

Where $\mathrm{d} 1=\operatorname{Sim} W \mathrm{p}(\mathrm{c} 1, \mathrm{vv} 1(\mathrm{c} 1))$ and $\mathrm{d} 2=\operatorname{SimWp}$ (c2,vrv2(c2)),

Example: we apply our structural method to make mapping between the two concepts Reformulate and Structure or design a course (Cf. Figure 8):

Let's $\mathrm{c} 1=$ Reformulate a course and $\mathrm{c} 2=$ Structure or design a course.

We suppose that SimS (explanatory annotations, analytic annotations) are similar with $\operatorname{SimS}=0.87$. We can distinguish two cases.

Case-1: $(\mathrm{c} 1, \mathrm{c} 2)=($ reformulate a course, structure a course $)=\{($ annotation explanatory, annotation analytical) $\}$;

$d l=$ SimWP (explanatory annotations, reformulate course) $=0.85$

$d 2=$ SimWP (analytical annotations, structure a course $)=0.88$

SimWP (reformulate a course, a course structure $)=$ 0.9 ;
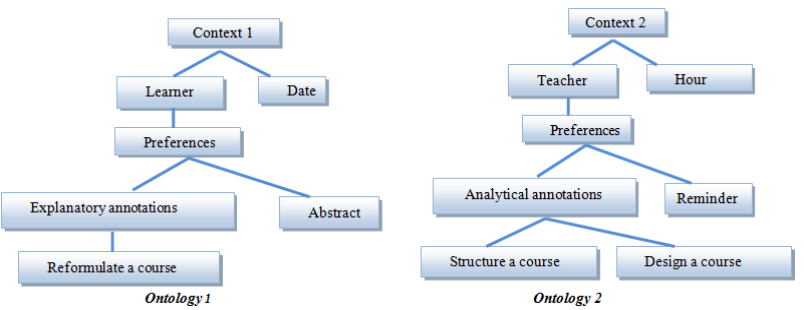

Figure 8. Example of our measurement method

And finally

SimStr $($ rephrase course, course structure $)=0.78$

Case-2: $(\mathrm{c} 1, \mathrm{c} 2)=($ reformulate a course, design course $)$ $=\{$ (explanatory annotations, analytical annotations $)\} \mid \mathrm{VR}$ $(\mathrm{c} 1, \mathrm{c} 2) \mid=1$;

$d 1=$ SimWP (explanatory annotations, reformulate course $)=0.85$

$d 2=$ SimWP (analytical annotations, design a course) $=0.8$;

$\operatorname{Sim} W P($ reformulate a course, design course $)=0.7$;

And finally

SimStr (reformulate a course, design course) $=0.6$

As a conclusion, we can deduce from these calculations that the similarity between (reformulate a course, a course structure) is more adequate than that between (reformulate a course, design a course).

\section{3) Calculation of global similarity}

We quantify the semantic similarity and the structural similarity in order to obtain the global similarity. Our aim is to align the ontology of the learning context and the ontology of annotation in order to provide the adequate learning for the current learning context. We seek a global similarity in order to optimize the learning quality as well as our annotation memory. The calculation of the similarity could be also used between the context of ontologies in the case of reusing the same learning context or searching for similar contexts in the context server, etc

Global Similarities Algorithm INPUT:

1) O1 and O2: Ontology 1 and Ontology 2

2) VSS: Semantic vector of similarities

3) VSSt: structural vector of similarities

4) WSimSt: Weight of structural similarities

5) WSimS: Weight of semantic similarities

OUTPUT:

VSG: global (Semantic and structural) vector of Similarities Begin

* go to each concept of ontology $1 *$ / For each ( $\mathrm{CO} 1 \varepsilon \mathrm{O} 1)$ do

/* go to each concept of ontology $2 * /$

For each $(\mathrm{CO} 2 \varepsilon \mathrm{O} 2)$ do If CO1.type $==\mathrm{CO} 2$.type then

*Extract semantic similarities between $\mathrm{CO} 1$ and $\mathrm{CO} 2$ of VSS*/ SimS=EXTRACTSIM (VSS, CO1, CO2)

*Extract structural simiraties between $\mathrm{CO} 1$ and $\mathrm{CO} 2$ of VSSt */

$\mathrm{SimSt}=$ EXTRActSIM (VSSt, CO1, CO2)

$/ *$ calculate global similarity*/ SimG $=$ SimS + SimSt

/* Add CO1, CO2 and SimG in VSG*/ Add ((CO1, CO2, SimG), VSG) Return (VSG)

Figure 9. Global similarity algorithm 


\section{CONCLUSION AND FUtURE WORK}

This paper represents a study to realize an adaptive annotation memory for context-aware training for the various actors in a CEHL. This memory can satisfy the need for training according to a given objective and a given context of all the actors in terms of utility, re-use, sharing and adaptability. ARAMAC is based on ontology engineering.

The running mechanism of our framework articulates around a whole of modules. Each module allows a functional need well defined and is composed of Web services. This framework aims to facilitate its integration, its interoperability with other e-learning systems.

The generic context ontology allows to formalize the proposed context data delivered by providers of context, these data are used by web services of context-aware learning through annotation memory to adapt the learning provided in a current context envisaging subsequent reuse. Thanks to the context, the annotation memory is aware (context-aware), which means it is on one side at every moment conscious of the context state and on the other hand it is sensitive to learning context changes by adapting the memory contents and functionality to the needs of all actors.

For this, we have formally described ontology with OWL to facilitate its integration into our learning based annotations memory.

As a perspective, we may also automatically enrich the learning context ontology by adding detailed classes that inherit from existing classes. This enrichment can be done by tracking different learning contexts of the different actors. As well, the services development for monitoring learning contexts, can help us to enrich the generic context ontology. We also believe that the addition of an ontology for the automatic deduction of the annotation context reuse according to each actor will provide an important enrichment to learning based annotations memory and its functionalities. This deduction will be based on the proposed generic context ontology.

We developed the generic ontology of pedagogical objective in order to implement it in our annotations memory dedicated to all actors. For this we describe the ontology with OWL.

As a perspective, we can develop a module for automatic enrichment of the generic ontology of pedagogical objective. This enrichment can be followed by the annotations and learning activities produced by actors. Therefore, we can enrich the objective ontology, the semantic annotations, the learning context which improve and increase the quality of all the learning process

In this paper, we have also describing our process of reuse of two levels for the annotation as a first level and then for the learning contexts as a second level, that is to say the manner in which this knowledge will be exploited in order to generate automatically a learning relatively adapted to a given pedagogical goal in a current context, in addition to the reuse of these contexts later. The reuse and capitalization in our approach are based on ontology alignment; we present thus our method of semantic, structural and global similarity measurements.

Several perspectives are possible, for this work. In Particular, We Aim to experiment and evaluate our method of calculation Similarities by adding improvements especially in terms of measurement functions that can give us better results.

\section{REFERENCES}

[1] 1. Marie-Hélène Abel, Mémoire d'Habilitation à diriger des Recherches, Apport des Mémoires Organisationnelles dans un contexte d'apprentissage, 2008

[2] 2. D. Mille 2005, Thèse., in Département informatique, Université Joseph- Fourier, Modèles et outils logiciels pour l'annotation sémantique de documents pédagogiques: Grenoble.

[3] Aksit, M., et Z. Choukair. 2003. « Dynamic, Adaptive and Reconfigurable Systems Overview and Prospective Vision ». In Proceedings of the ICDCSW'03 (May, 2003). p. 84-92. Providence, Rhode Island, USA.

[4] C. Desmoulins and F. Azouaou, Taking Teaching Context into Account for Semantic Annotation Patterns.,EC-TEL2006 pp. 543548, 2006.

[5] Ouadah, F. Azouaou, and C. Desmoulins, 2009, 2ème conférence internationale sur l'informatique et ses applications, CIIA09, Modèles et Architecture d'une Mémoire d'Annotation context-aware pour l'Enseignant., 03-04 Mai 2009, Saida, Algérie.

[6] Schmidt, Bridging the Gap between Knowledge Management and ELearning withContext-Aware Corporate Learning. in Third Biennial Conference, WM 2005.Revised Selected Papers, Lecture Notes in Artificial Intelligence (LNAI). 2005. Kaiserlautern,Germany: Springer.

[7] Mille D., Thèse., in Département informatique, Université JosephFourier, Modèles et outils logiciels pour l'annotation sémantique de documents pédagogiques: Grenoble. p. 173 pages

[8] D. Howe, Free online dictionary of computing. London, UK, Imperial College Departement of Computing, last access date 12.02.2011.

[9] A. Dey, Providing Architectural Support for Building ContextAware Applications, in College of

[10] Computing. 2000, Georgia Institute of Technology, pp 23-35, last access date 05.01.2011.

[11] Chen, G. et Kotz, D. A Survey of Context-aware Mobile Computing Research . Technical Report: TR2000-381 Dartmouh College. Hanover, NH, USA.p.16.2000

[12] Schilit B.N., Adams N.I., Want R., "Context-Aware Computing Applications", Proceedingsof the IEEE Workshop on Mobile Computing Systems and Applications (WMCSA'94), IEEE Press, p 85-90.

[13] Chaari T. "Adaptation d'applications pervasives dans des environnements multi-contextes". PhDthesis a l'institut national des sciences appliquees de lyon, laboratoire LIRIS, 2007

[14] Hakim Mokeddem, Faiçal Azouaou, Cyrille Desmoulins, 2009, CIIA 2009: Ontologie de la Sémantique de l'Annotation Pédagogique de l'Apprenant.

[15] IEEE, IEEE Standard for Learning Object Metadata, 2007, Learning Technology Standards Committee.

[16] Design, I.L. IMS Learning Design. 2005, Available from, http://www.imsglobal.org/learningdesign.

[17] N. Duclosson, S. Jean-Daubias, and S. Riot, AMBRE- enseignant: un module partenaire de l'enseignant pour créer des problèmes, EIAH'2005, Montpellier, 25-27 mai 2005, pp. 353-358.

[18] P. Leroux, Machines partenaires des apprenants et des enseignants - Étude dans le cadre d'environnements supports de projets pédagogiques. Habilitation à Diriger des Recherches, Université du Maine, 2002,

[19] S. Girard and H. Johnson, DividingQuest: opening the learner model to teachers, EIAH'2007, Lausanne, 27-29 juin 2007, pp. 329-334,

[20] M. Lefevre, S. Jean-Daubias, and N. Guin. Personnaliser des séquences de travail à partir deprofils d'apprenants, EIAH 2009, Le Mans, 23-26 juin 2009, pp. 1136-1141.

[21] M. Miraoui, C. Tadj, C. Ben Amar, "Context-Aware Services Adaptation in a Pervasive Computing System", The Third International Conference on Mobile Ubiquitous Computing, Systems, Services and Technologies. UBICOMM 2009.

[22] Nadia ALOUI, Faiez GARGOURI, Ontology-based Architecture for Reusing and Learning Through Context-aware Annotations Memory, ICCGIS, IARIA conference, Luxemburg 2011. 
PAPER

OARLCAM: AN ONTOLOGY-BASED APPROACH FOR REUSING AND LEARNING THROUGH CONTEXT-AWARE ANNOT...

[23] Noy N., " Semantic Integration: A Survey of Ontology-based Approaches », SIGMOD, 2004.

[24] Derntl, M. and K.A. Hummel. Modeling Context-Aware e- Learning Scenarios. in 3rdIEEE International Conference on Pervasive Computing and Communications. 2005. Kauai Island: IEEE Computer Society.

[25] Nadia ALOUI, Faiez GARGOURI, Generic context ontology for a Learning Through Context-aware Annotations Memory, ICICS, ACM international conference, Jordan, 2012.

[26] Nadia ALOUI, Faiez GARGOURI, a generic ontology of objective for learning through a context-aware annotations memory, IADIS e-learning conference, Portugal, 2012.

[27] Nadia ALOUI, Faiez GARGOURI, An ontolgy-based approach for learning annotations reuse, ICEELI, IEEE International conference, Sousse, 1-3 juillet 2012.

[28] W. Cohen R . Pradeep Ravikumar, Stephen E. " A Comparison of String Distance Metrics for Name-Matching Tasks ",American Association for Artificial Intelligence,2003.
[29] Aissa Fellah, Mimoun Malki, Ahmed ZAHAF, Alignement des ontologies: utilisation de WordNet et une nouvelle mesure structurelle, Conférence en Recherche d'Information et Applications, CORIA 2008.

[30] H. Abolhassani H. , B.B. Hariri, S. H. Haeri, "On Ontology Alignment Experiments", Webology, Volume 3, Number 3, September, 2006.

[31] S. Zghal, Contributions à l'alignement d'ontologies OWL par agrégation de similarités. Thèse de doctorat. Université de Tunis. 23 Octobre 2010.

[32] T. Touzani M., " Alignement des ontologies OWL-Lite », Master's thesis, University of Montreal, 2005

\section{AUTHORS}

N. Aloui and F. Gargouri are with the MIRACL Laboratory at University of Sfax, Sfax, Tunisia.

Submitted 02 August 2013. Published as resubmitted by the authors 14 September 2014. 